\title{
Molecular Insights into X;BTA5 Chromosome Rearrangements in the Tribe Antilopini (Bovidae)
}

\author{
H. Cernohorska ${ }^{a}$ S. Kubickova ${ }^{a} \quad$ J. Vahala ${ }^{b} \quad$ J. Rubes $^{a}$ \\ ${ }^{a}$ Veterinary Research Institute, Brno, and ${ }^{b}$ Zoo Dvur Kralove, Dvur Kralove n. L., Czech Republic
}

\section{Key Words}

Antilopini - Chromosome painting · Fluorescence in situ hybridization - Fusion site $\cdot$ Nucleolus organizer region • Satellite DNA $\cdot \mathrm{X}$-autosome translocation

\begin{abstract}
For a clade that includes Antilope, Gazella, Nanger and Eudorcas (Antilopinae), $\mathrm{X} ; \mathrm{BTA} 5$ translocation is a synapomorphy. Using a combination of fluorescence in situ hybridization (FISH) probes and polymerase chain reaction techniques, we provide (i) the first insight into the $\mathrm{X} ; \mathrm{BTA5}$ architecture which differs in the species under study: Antilope cervicapra (genus Antilope), Gazella leptoceros (genus Gazella) and Nanger dama ruficollis (genus Nanger), (ii) determination of interstitial satellite DNA at the X;BTA5 junctions, and (iii) determination of repetitive sequences occupying constitutive heterochromatin of Xp arms in the studied species. The distribution of 2 repetitive DNA families in the centromeric regions of all chromosomes has been investigated by FISH with probes representing satellite I and satellite II DNA in all studied species. In this context, we discuss a markedly smaller centromere in the BTA5 (Y2) unfused chromosomes in males in the XY1Y2 determining system in comparison with other acrocentrics. An analysis of karyotypic data described in current published studies revealed a disparity with the data determined by FISH. In this report, we document chromosomal fusions in the 3 species mentioned
\end{abstract}

\section{KARGER}

Fax +4161306 1234

E-Mail karger@karger.ch

www.karger.com
(C) 2012 S. Karger AG, Basel

$1424-8581 / 12 / 1363-0188 \$ 38.00 / 0$

Accessible online at:

www.karger.com/cgr resulting from FISH with painting probes prepared from cattle (Bos taurus). The number and chromosomal location of nucleolus organizer regions were determined by $\mathrm{FISH}$. In the present study, we emphasize the importance of chromosomal rearrangement verification, particularly, if they are used for phylogenetic analysis.

Copyright $\odot 2012$ S. Karger AG, Basel

One of the most controversial mammalian taxa from a classification point of view is that of the family Bovidae being subdivided into 2 distinct clades - Bovinae (Bovini, Boselaphini and Tragelaphini) and Antilopinae (Aepycerotini, Alcelaphini, Antilopini, Caprini, Cephalophini, Hippotragini, Neotragini, Oreotragini and Reduncini). The most recent classification recognizes 9 genera within Antilopini: Ammodorcas, Antidorcas, Antilope, Eudorcas, Gazella, Litocranius, Nanger, Procapra and Saiga [Robinson and Ropiquet, 2011].

Chromosomal evolution in Bovidae is largely attributable to Robertsonian fusions, although the increasing number of cytogenetic reports reveals that tandem fusions also impact karyotype shaping in this family [Rubes et al., 2008; Ropiquet et al., 2010; Cernohorska et al., 2011] by reducing the ancestral diploid number $(2 n=60)$ in derived karyotypes. Many Robertsonian chromosomal fusions are homoplasic, or are often lineage-specific (autapomorphic), and thus phylogenetically uninformative [Robinson and 
Ropiquet, 2011]. In contrast, a great variety of X chromosomes including changes in centromere placement, multiple transpositions of common segments, heterochromatic variation and autosomal translocation have been evolved within Bovidae [Robinson et al., 1998; Iannuzzi et al., 2000; Chaves et al., 2004; Rubes et al., 2008]. Thus, a similarity in $\mathrm{X}$ chromosome appearance may provide a rich source of phylogenetic information in this family. For example, an inversion of the $\mathrm{X}$ chromosome defines the monophyly of Bovinae or an X centromeric shift defines the Bovina [Robinson and Ropiquet, 2011]. Antilope, $G a-$ zella, Nanger and Eudorcas may form a clade within Antilopini sharing the sex chromosome/autosome translocation (X;BTA5). Chromosomal rearrangements involving the gonosomes can negatively affect fertility in male carriers [Ashley, 2002]. On the other hand, their relatively widespread occurrence among mammals [Dobigny et al., 2004] indicates that this rearrangement is meiotically acceptable.

In the present investigation we document the outcome of molecular cytogenetic dissection of the X;BTA 5 compound chromosomes, nucleolus organizer region (NOR) locations, distribution of 2 repetitive DNA families in the centromeric regions of all chromosomes and revision of the previously published chromosomal fusions in 3 members of the studied clade: Antilope cervicapra $(2 \mathrm{n}=30-$ 33), Gazella leptoceros $(2 \mathrm{n}=32$ \% $33 \hat{\jmath})$ and Nanger dama ruficollis $(2 \mathrm{n}=38-40)$. Our findings highlight the importance of using molecular methods, particularly if the outcomes are used for phylogenetic studies.

\section{Animals and Methods}

\section{Animals}

Peripheral blood samples of the following captive-born specimens were used: A. cervicapra (blackbuck, 2 females and 2 males, origin unknown) held in the Plzen Zoological Garden (Czech Republic), a $N$. d. ruficollis (dama gazelle) male and a G. leptoceros (rhim gazelle) male held in the Dvur Kralove (Czech Republic) animal collection. According to the Zoo's animal records, these captive specimens have long been bred in captivity and are of unknown origin.

\section{Culture Conditions and G-Banding}

Whole blood $(2.7 \mathrm{ml}$ ) was added to $22 \mathrm{ml}$ of RPMI 1640 (Sevapharma, Prague, Czech Republic) culture medium supplemented with $20 \%$ of fetal calf serum (Sigma-Aldrich Corp., St. Louis, Mo., USA), glutamine $(0.5 \mathrm{mg} / \mathrm{ml}$, Sevapharma), pokeweed mitogen $(0.15 \mathrm{mg} / \mathrm{ml}$, Sigma) and antibiotic-antimycotic solution (Gibco, Grand Island, N.Y., USA). The cultures were harvested after $72 \mathrm{~h}$ of incubation at $38^{\circ} \mathrm{C}$. Colcemid (Sigma) was added to the cultures at a final concentration of $0.1 \mu \mathrm{g} / \mathrm{ml}, 40 \mathrm{~min}$ before the end of the incubation.

Molecular Insights into X;BTA5

Compound Chromosomes
Harvested cells were exposed to a 20-min hypotonic treatment in $0.56 \%$ KCL followed by ethanol:acetic acid (3:1) fixation. Gbanding [Seabright, 1971] was performed on metaphase chromosomes [Verma and Babu, 1989] with a slight modification. Before trypsin treatment, slides were aged at $60^{\circ} \mathrm{C}$ for $2 \mathrm{~h}$, and then incubated in $2 \times \mathrm{SSC}$ in a $60^{\circ} \mathrm{C}$ water bath for $30 \mathrm{~min}$. G-banded chromosomes were numbered according to the Bos taurus standard [ISCNDB 2000].

Chromosome Painting and Fluorescence in situ Hybridization

We used the PALM Microlaser system (Carl Zeiss MicroImaging $\mathrm{GmbH}$, Munich, Germany) for the generation of whole cattle chromosome painting probes (BTA1-BTA29, Kubickova et al. [2002]) for cross-species hybridization to A. cervicapra, N. d. ruficollis and G. leptoceros chromosomes to examine their chromosomal fusions. In order to determine the type of $\mathrm{X}$ chromosomes in A. cervicapra, N. dama ruficollis and G. leptoceros, we prepared arm-specific BTAXp and BTAXq painting probes, as well as region-specific BTAXq3.6-qter and CHIXq4.1-qter (Capra hircus) probes. Additionally, a subchromosomal probe from the terminal end of BTA5 was constructed permitting the orientation of the $\mathrm{X}$;BTA5 translocations. The microdissected NDRXp painting probe enabled us to confirm the inverted position of the sexual part of ACEX. All probes were labeled with Spectrum Orange-dUTP and Spectrum Green-dUTP (Abbott, Abbott Park, Ill., USA).

The hybridization mixture $(10 \mu \mathrm{l})$ for double-color fluorescence in situ hybridization (FISH) containing $50 \%$ formamide, $2 \times$ SSC, $10 \%$ dextran sulphate, $7 \mu \mathrm{g}$ of salmon sperm DNA, 1.3 $\mu \mathrm{l}$ of bovine Hybloc ${ }^{\mathrm{TM}}$ DNA ( $1 \mathrm{mg} / \mathrm{ml}$ ) (Applied Genetics Laboratories, Melbourne, Fla., USA) and $1.5 \mu$ l of differently labeled probes were denatured at $72^{\circ} \mathrm{C}$ for $10 \mathrm{~min}$ and preannealed at $37^{\circ} \mathrm{C}$ for $60 \mathrm{~min}$. Metaphase spreads were denatured in $70 \%$ formamide, $2 \times \mathrm{SSC}(\mathrm{pH} 7.0)$ at $72^{\circ} \mathrm{C}$ for $2 \mathrm{~min}$, dehydrated through cold alcohol series (70\%, 80\% and absolute ethanol) and hybridized for $24 \mathrm{~h}$ in a moist chamber at $37^{\circ} \mathrm{C}$. Slides were washed in $0.4 \times \mathrm{SSC}\left(\mathrm{pH} \mathrm{7.0)}\right.$ ) at $72^{\circ} \mathrm{C}$ for $2 \mathrm{~min}$, counterstained with 4',6-diamidino-2-phenylindole (DAPI) and mounted in Vectashield mounting medium (Vector Laboratories, Burlingame, Calif., USA). Hybridization signals were examined using an Olympus BX 60 fluorescent microscope and digital images were captured with ISIS 3 software (MetaSystems, Altlussheim, Germany). When the same slide was used in subsequent hybridization experiments, the coverslip was gently removed and the slide washed in a buffer containing $0.05 \%$ Tween (Serva Electrophoresis GmbH, Heidelberg, Germany), dehydrated and reused.

For generation of sat I and sat II centromeric clones from $N$. $d$. ruficollis, G. leptoceros and A. cervicapra, DNA templates were taken from centromeric regions of 1 animal per species, respectively, by laser microdissection, amplified by degenerate oligonucleotide primed polymerase chain reaction (PCR) and subsequently cloned into a pDrive vector (Qiagen, Hilden, Germany). Species-specific clones were selected by DOT BLOT hybridization following Pauciullo et al. [2006], fluorescently labeled and checked by FISH. Subsequently, plasmid DNA was isolated and sequenced. Sequences comprised satellite DNA but were not long enough to represent the whole basic repeat unit. Therefore, according to the sequence of centromere-specific clones, appropriate primers for amplification of $5^{\prime}$ - and $3^{\prime}$-flanking regions have been designed and inverse PCR was performed on the whole genome DNA to obtain the basic repeat unit of satellite DNAs. The amplification 
products were cloned and then plasmids were isolated and sequenced using BigDye terminator chemistry on an automated sequencer. We characterized several centromeric clones isolated from 1 animal per species representing the main motifs of the repetitive DNA. The sequences were compared to the GenBank database using BLASTN and BLAST2 searches.

Detection of Satellite DNA in X;5 Junctions by PCR

We probed for the presence of interstitial satellite DNA (centromeric repeats) at the X;BTA5 junctions in ACE, NDR and GLE chromosomes using PCR procedures and the following approach. DNA regions representing a fusion between $\mathrm{X}$ and autosome BTA5 were microdissected (each fusion site in the 3 species was microdissected separately). Additionally, in each species, the random mix of centromeric regions and the distal parts of different autosomes were used as PCR controls (positive and negative, respectively) in these experiments. The dissected regions mentioned above were collected and used as template DNA for PCR with 2 different primer pairs: (a) first pair, termed SI, designed from the coincident sequences of all sat I clones: $5^{\prime}$-TCAAGGCAGTGCAGGGCAGTCAGG-3' and 5'-ACTTATCCAATGAGGCGTCTTGGG-3' amplifying a 357-bp product, and (b) the second pair, termed SII, derived from the coincident sequences of all sat II clones: $5^{\prime}$-TCTGCTCAGAGGCCCGATCTGG-3' and 5'-CAAGGCAGGAGCGGAGCTGAGG-3' amplifying a 167-bp product. Cycling parameters were $95^{\circ} \mathrm{C}$ for $4 \mathrm{~min}$ for initial denaturation, 30 cycles at $95^{\circ} \mathrm{C}$ for $1 \mathrm{~min}, 61^{\circ} \mathrm{C}$ for $1 \mathrm{~min}$, and $72^{\circ} \mathrm{C}$ for $2 \mathrm{~min}$, with a 5 -min final extension at $72^{\circ} \mathrm{C}$. Two rounds of the same PCR were performed and amplification products were characterized by gel electrophoresis on a $2 \%$ agarose gel.

\section{Preparation of the Xp-Specific Probe (Xph Clone)}

This probe was constructed by laser microdissection of the heterochromatic ACEXp arms. Procedures for amplification, labeling, cloning, DOT BLOT analysis and sequencing were performed as described above. Subsequently, the clone we termed Xph was hybridized to metaphase chromosome spreads of the $A$. cervicapra, N. d. ruficollis and G. leptoceros to determine their FISH signals on the chromosomes.

Additionally, we separately collected the Xp arms of N.d. ruficollis and A. cervicapra (used as positive PCR controls) for PCR procedures with the Xph-specific primers 5'-GAGACACTGTTCCCAGAAGCAGG-3' and 5'-GTGACCATATTTCCATCTGAGTGG-3', which were designed from the Xph sequence to probe for the presence of these repetitive sequences in $N$. d. ruficollis. Cycling parameters were the same as described above, except for the primer annealing temperature of $58^{\circ} \mathrm{C}$. The obtained PCR products were labeled with SpectrumOrange-dUTP by another round of PCR. These were subsequently used for confirmation of their homology with the original Xph clone by hybridization to ACE and NDR chromosomes.

\section{Preparation of the NOR Identifying Probe}

NORs were visualized by silver staining on $N$. d. ruficollis chromosomes followed by the standard techniques described by Goodpasture and Bloom [1975]. Subsequently, on the same chromosomes, a C-banding technique was done following the standard protocol of Sumner [1972] to eliminate unique DNA sequences of chromosomes. After this treatment, we microdissected the telomeric region of autosome BTA5 bearing the NOR
Table 1. Centric and tandem chromosome fusions in 3 species of Antilopini: blackbuck (Antilope cervicapra), dama gazelle (Nanger dama ruficollis), rhim gazelle (Gazella leptoceros)

\begin{tabular}{lll}
\hline Blackbuck & Dama gazelle & Rhim gazelle \\
\hline $\mathrm{t}(1 ; 25)$ & $\mathrm{t}(2 ; 15)$ & $\mathrm{t}(2 ; 3)$ \\
$\mathrm{t}(2 ; 29)$ & $\mathrm{t}(4 ; 28)$ & $\mathrm{t}(4 ; 6)$ \\
$\mathrm{t}(3 ; 27)$ & $\mathrm{t}(6 ; 25)$ & $\mathrm{t}(7 ; 21)$ \\
$\mathrm{t}(4 ; 19)$ & $\mathrm{t}(7 ; 22)$ & $\mathrm{t}(8 ; 14)$ \\
$\mathrm{t}(6 ; 24)$ & $\mathrm{t}(8 ; 21)$ & $\mathrm{t}(9 ; 17)$ \\
$\mathrm{t}(7 ; 20)$ & $\mathrm{t}(9 ; 26)$ & $\mathrm{t}(10 ; 20)$ \\
$\mathrm{t}(8 ; 14)$ & $\mathrm{t}(10 ; 23)$ & $\mathrm{t}(11 ; 27)$ \\
$\mathrm{t}(9 ; 17)$ & $\mathrm{t}(12 ; 27)$ & $\mathrm{t}(12 ; 16)$ \\
$\mathrm{t}(10 ; 28)$ & $\mathrm{t}(13 ; 17)$ & $\mathrm{t}(13 ; 19)$ \\
$\mathrm{t}(11 ; 22)$ & $\mathrm{t}(14 ; 24)$ & $\mathrm{t}(18 ; 29)$ \\
$\mathrm{t}(12 ; 16)$ & & $\mathrm{t}(22 ; 25)$ \\
$\mathrm{t}(13 ; 18)$ & & $\mathrm{t}(23 ; 24)$ \\
$\mathrm{t}(15 ; 23)$ & & $\mathrm{t}(26 ; 28)$ \\
$\mathrm{t}(21 ; 26)$ & & $\mathrm{t}(\mathrm{X} ; 5)$ \\
$\mathrm{t}(\mathrm{X} ; 5)$ & $\mathrm{t}(\mathbf{X} ; 5)$ & $\mathrm{Y}$ \\
$\mathrm{Y}$ & $\mathrm{t}(\mathrm{Y} ; 16)$ &
\end{tabular}

Chromosomes were numbered according to the standard karyotype of cattle (Bos taurus) presented by the ISCNDB 2000. Bold numbers indicate tandem fusions.

region, amplified and labeled the sequences with SpectrumOrange-dUTP and used them as a FISH probe on NDR, GLE, and ACE chromosomes.

\section{Results}

\section{Karyotype Description}

Homologies of different arms of centric fusions with bovine autosomes were identified by G-banding and subsequently verified by painting probes derived from cattle. The hybridization results are shown in table 1 . Y chromosomes of $A$. cervicapra and $G$. leptoceros are acrocentric, whilst the Y chromosome in $N$. d. ruficollis is submetacentric and is connected by centric fusion to BTA16. All the NORs are located in the telomeric regions of chromosomes corresponding to BTA2-BTA5 in A. cervicapra and G. leptoceros (fig. 1a). In N. d. ruficollis, NORs are located on BTA2, BTA4 and BTA5 (fig. 1b).

\section{$X$ Chromosomes}

The studied species exhibit an X-autosomal translocation involving BTA5, resulting in an imbalance in diploid numbers between sexes (i.e. XY1Y2 sex chromosome system). The sex-autosome fusion products can be classified into these morphological types: 

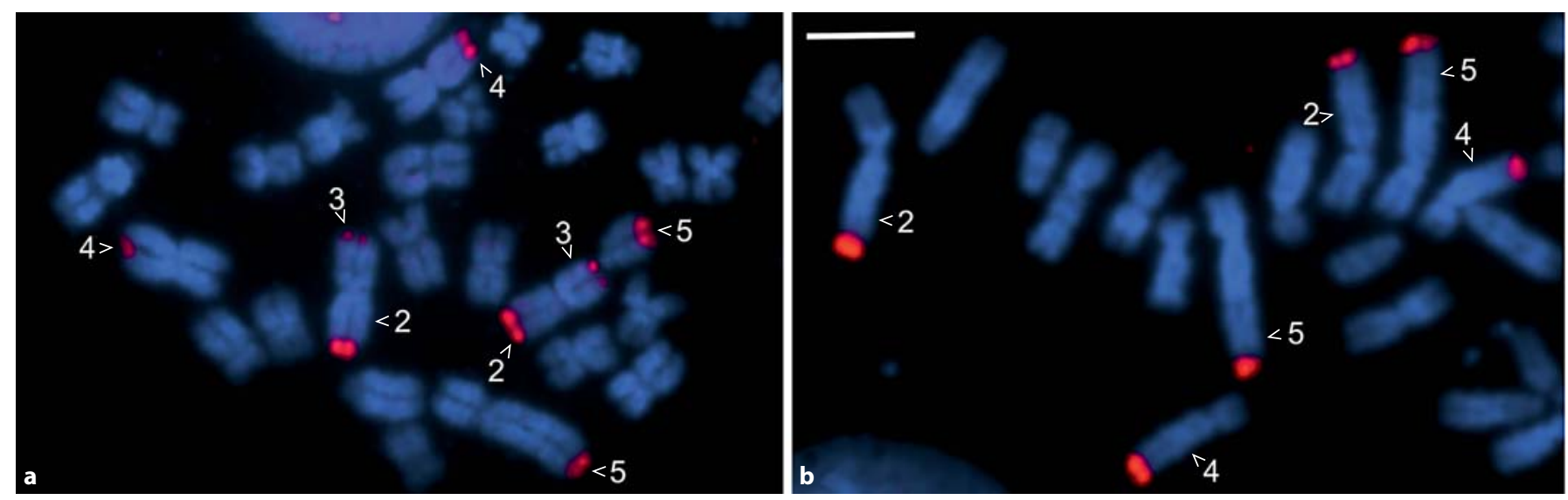

Fig. 1. NOR location in a Gazella leptoceros and $\mathbf{b}$ Nanger dama ruficollis identified by a fluorescently labeled probe prepared from BTA5 telomeric regions of $N$. $d$. ruficollis chromosomes by laser microdissection. Bar $=5 \mu \mathrm{m}$.

(i) Submetacentric chromosome with the caprine type of X fused with BTA5 by tandem fusion (tail-to-head). The centromere is inserted approximately in the middle of the sexual part of the $\mathrm{X}$ chromosome. This type of $\mathrm{X}$ has been found in N. d. ruficollis (fig. 2a, b). We looked for interstitial centromeric repeats at the junction point between chromosomes X and BTA5 by PCR, using 2 primer pairs (SI and SII). The results showed positive PCR amplifications with both primer pairs, indicating the presence of sat I and II DNAs at the X;BTA5 junction site (fig. 2d). At this site, hybridization signals were detected as well, using fluorescently labeled sat I and II clones (fig. 3).

(ii) Submetacentric chromosome with the caprine type of X fused with BTA5 by tandem fusion. The Xp arm is entirely heterochromatic and short. This type of $\mathrm{X}$ has been found in G. leptoceros (fig. 2a, b). PCR procedures with the SI and SII primer pairs and the microdissected $\mathrm{X}$;BTA5 junction region used as DNA template revealed only the presence of sat I sequences at this point (fig. 2d). When hybridized with sat I and II probes, no labeling was detected at the junction.

(iii) Submetacentric chromosome with a caprine type of X fused with BTA5 by centric fusion (head-to-head). This type of $\mathrm{X}$ has been found in A. cervicapra. The $\mathrm{X}$ chromosome is in an inverted position with a shift in centromere position to the terminal (telomeric) end of the sexual part of the X (fig. 2a, b). The fluorescent signals obtained from the NDRXp (see Materials and Methods) painting probe showed hybridization to 2 regions of ACEX (fig. 2c). The signal approximately in the middle part of the X confirms both the inverted position of the sex segment of the $\mathrm{X}$ in ACE and its centric fusion between X and autosome BTA5. The signal on the ACEXp arms confirmed $\mathrm{Xp}$ repetitive sequences homologous with NDRX. The short ACEXp arms are entirely heterochromatic. PCR procedures with the 2 primer pairs (SI and SII) and microdissected head-to-head junction site between X and BTA5 chromosomes used as a DNA template gave no products (fig. $2 \mathrm{~d}$ ) suggesting either the $\mathrm{ab}$ sence of centromeric DNA or different repetitive sequences at this junction point.

\section{Xp Arms}

To study the constitutive heterochromatin of Xp arms, we isolated and cloned repetitive DNA dissected from $A$. cervicapra (clone $\mathrm{Xph}$ ) and hybridized it to the 3 species studied.

In the case of $A$. cervicapra, the Xph clone labeled Xp arms as well as the Y chromosome, with the exception of its centromere (fig. 4a). The centromere was labeled with ACE-specific sat I probe (fig. $5 \mathrm{c}$ ).

In G. leptoceros the Xph clone showed strong hybridization signals on the Xp arm and 2 separate blocks of the $\mathrm{Y}$ chromosome suggesting that the constitutive heterochromatin on the Xp arms of both species was formed by the same repeat component. Moreover, in this species, GLE-specific sat I clone hybridized to both Xp arms and the middle part of the $Y$, including its centromere (fig. $4 \mathrm{~b}$ ).

In $N$. d. ruficollis, the fluorescent signal of the Xph clone was restricted only to the sexual part of the Y chromosome. In this region, NDR-specific sat I DNA gave fluorescence as well (fig. 4c). When the Xp arms were analyzed by PCR with Xph-specific primers, positive PCR 

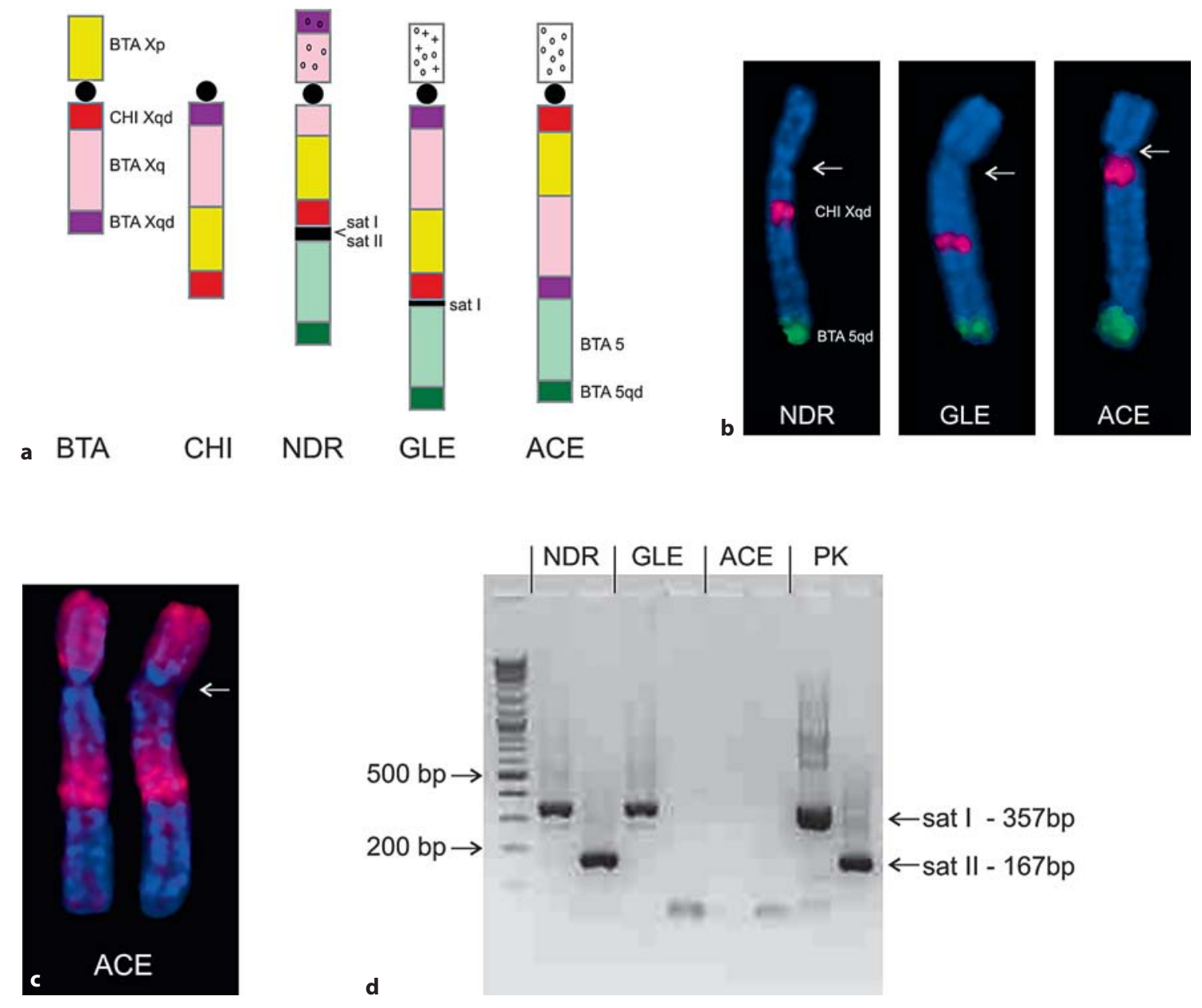

Fig. 2. a Schematic illustration showing chromosome homologies between 5 related species. Bos taurus (BTA), Capra hircus (CHI), N. d. ruficollis (NDR), G. leptoceros (GLE) and A. cervicapra (ACE). Region-specific painting probes are marked in different colors. Circles indicate the presence of Xp-specific repetitive DNA (Xph clone); plus signs indicate GLE-specific sat I DNA. b FISH images of subchromosomal painting probes permitting the orientation of the X;BTA5 fusions. Colors are coded as for the scheme. Arrows indicate the position of functional centromeres. c X chromosomes of A. cervicapra labeled with a painting probe prepared from Xp arms of $N$. d. ruficollis. The signal on Xp arms gives evidence of the presence of Xp-specific repetitive DNA in $\mathrm{Xp}$ arms of NDR (see schematic diagram) and the signal approximately in the middle of $\mathrm{Xq}$ demonstrates the centric fusion between $\mathrm{X}$ and BTA5 in A. cervicapra. Centromere is arrowed. $\mathbf{d}$ Gel showing satellite DNA patterns after PCR analysis with SI (detection of sat I DNA) and SII (detection of sat II DNA) primers and dissected X;BTA5 fusion sites as DNA template. PK = Positive control; molecular weights are represented on the first line. 


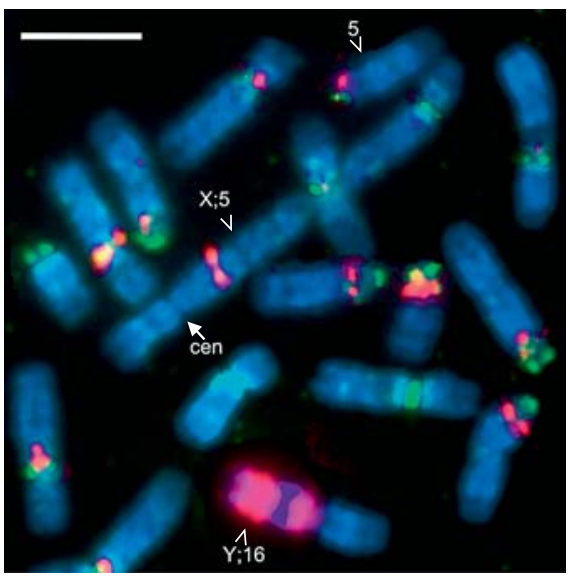

Fig. 3. Partial metaphase chromosomes of N. d. ruficollis after cohybridization with NDR-specific sat I (red, pericentric area) and sat II (green, centromeric area) probes. The functional centromere of X;BTA5 is shown by an arrowhead. Chromosomes are counterstained with DAPI (blue). Bar $=5 \mu \mathrm{m}$.

amplification was shown on the gel. We assumed that Xpspecific sequences were evenly dispersed in this area, but in an insufficient amount to be detected by FISH.

\section{Hybridizations with sat I and sat II Clones Derived}

from NDR, GLE and ACE Centromeres and Their Use for Comparative Analysis

We isolated and characterized centromeric clones representing the main motif of sat I and II of the 3 Antilopini species.

(i) In N. d. ruficollis, hybridization with NDR-specific sat I and II DNA clones resulted in different distribution patterns in the centromeric regions (i.e. centromeric and pericentric regions) of several chromosome pairs. The sat I probe gave strong fluorescence on 2 separate blocks of the male-specific part of the Y compound chromosome (Y;16) and X;BTA5 (tail-to-head) fusion site (fig. 3). Weaker signals were observed in pericentric regions of the majority of autosome pairs. The centromere of autosome BTA5 in males and the X;BTA5 functional centromere were clearly unlabeled (fig. 5a).

The sat II probe hybridized to centromeric regions of all chromosomes with an exception of Y;BTA16 (the translocation is characteristic of this species), X;BTA5 and acrocentric autosome BTA5 in males (fig. 5d). A weak signal was detected in the X;BTA5 junction site after $48 \mathrm{~h}$ of hybridization.

(ii) In G. leptoceros, GLE-specific sat I probe hybridized intensely to the $\mathrm{Y}$ chromosome (centromeric area and its middle part), on the short Xp arms and on the majority of autosome centromeres with an exception of autosome BTA5 in males (fig. 5b). The GLE-specific sat II probe labeled the centromere of the $\mathrm{X}$ and partly the $\mathrm{Xp}$ arm and 2 pairs of acrocentric autosomes (BTA1, BTA15; these chromosomes are not involved in Robertsonian fusions). Weaker signals were observed on several pairs of bi-armed chromosomes, no fluorescence was detected on Y, BTA5 in males and the rest of bi-armed autosomes (fig. 5e).

(iii) In A. cervicapra, hybridization with ACE-specific sat I probe resulted in a strong fluorescent signal in the centromeric regions of the unfused acrocentric autosomes BTA8 and BTA14 (these chromosomes are involved in centric fusion polymorphism) and the centromeric area of Y. Bi-armed chromosomes showed significantly weaker signals than the acrocentrics. The functional centromere of X;BTA 5 was unhybridized (fig. 5c). Hybridization with ACE-specific sat II gave fluorescence in all chromosomes, including the centromere of the $\mathrm{X}$, with an exception of the Y. No fluorescence was detected in autosome BTA 5 in males using both probes (fig. 5f).

According to BLAST2 searches, no sequence homology was found between sat I and sat II DNAs in all 3 studied species. The sequence similarity among the different clones of sat I DNAs (the clone length was about $790 \mathrm{bp}$ ) isolated from 1 animal per species was $73-85 \%$. The interspecies homology of sat I repeat units ranged between 72 and $93 \%$ (the highest homology of $93 \%$ was between A. cervicapra and G. leptoceros). With regard to the sat II clones (length about $700 \mathrm{bp}$ ), the BLAST2 analysis showed $71-96 \%$ homology in individual animals. The interspecies homology was 71-86\%, the highest homology (86\%) was again between A. cervicapra and G. leptoceros. In addition, we found that $70 \%$ of interspecies homology was sufficient for successful cross-species fluorescent hybridization.

\section{Discussion}

\section{Karyotype Description by FISH}

Our results showed that karyotypes of different wild animals in the studies based on banding techniques contained inaccuracies and should be verified by molecular methods, particularly, if they are used for phylogenetic analysis. The identification of small bovid autosomes is particularly difficult.

Karyotype descriptions in N.d. ruficollis and G. leptoceros were previously compiled by Vassart et al. [1995] and 

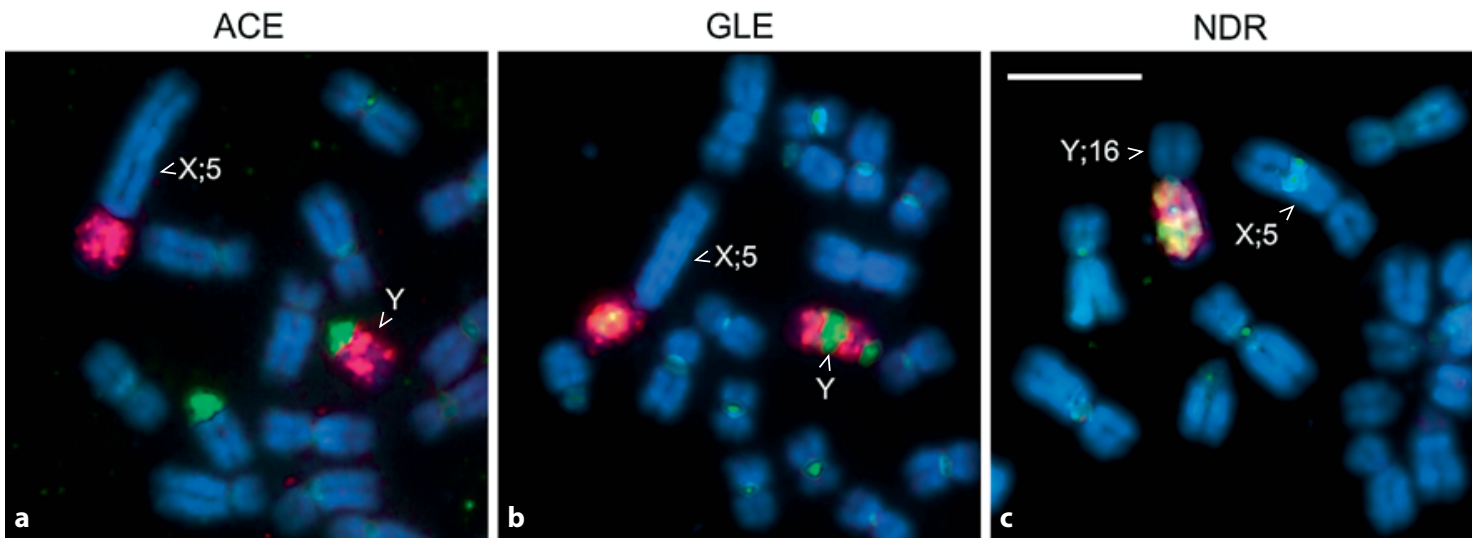

Fig. 4. The 3 panels show partial metaphase chromosomes after cohybridization with Xp-specific repetitive DNA (Xph clone, red) and species-specific sat I probes (green) in: a A. cervicapra, b G. leptoceros, c N. d. ruficollis. In this species, the sexual part of the $\mathrm{Y}$ is occupied by both sequences resulting in a yellow color on the chromosome. Chromosomes are counterstained with DAPI (blue). Bar $=5 \mu \mathrm{m}$.
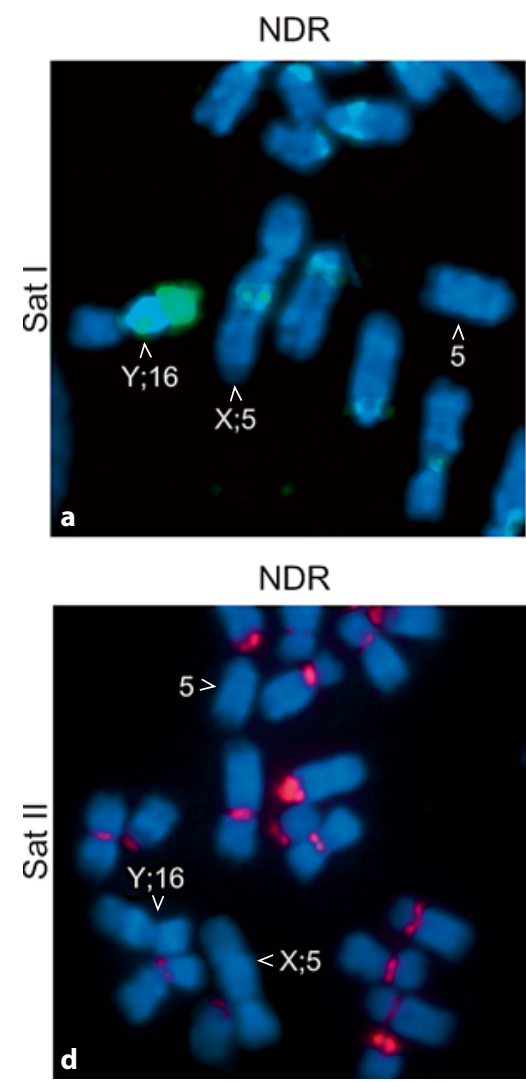

GLE

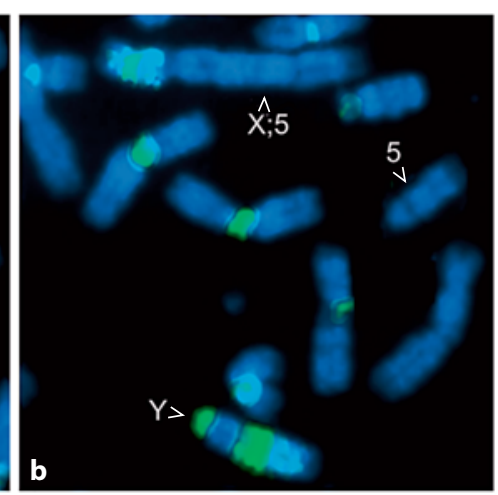

GLE

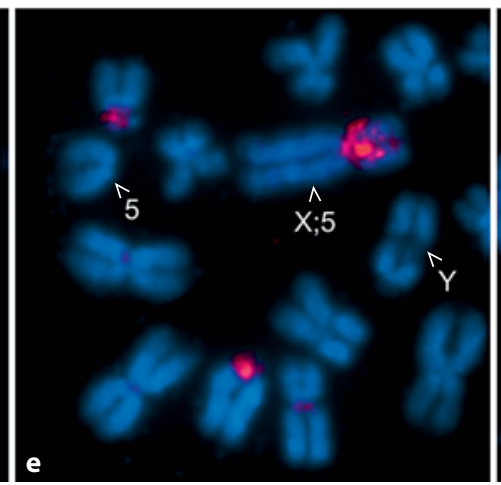

ACE

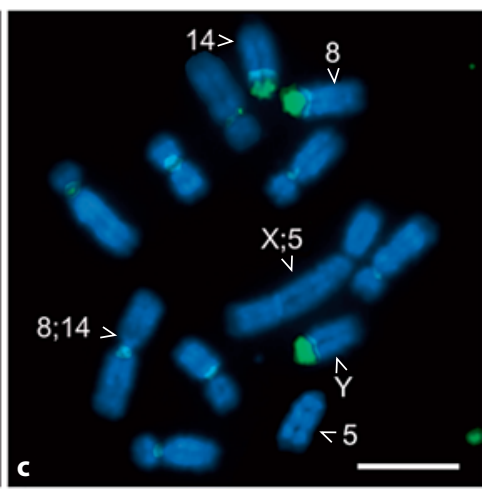

ACE

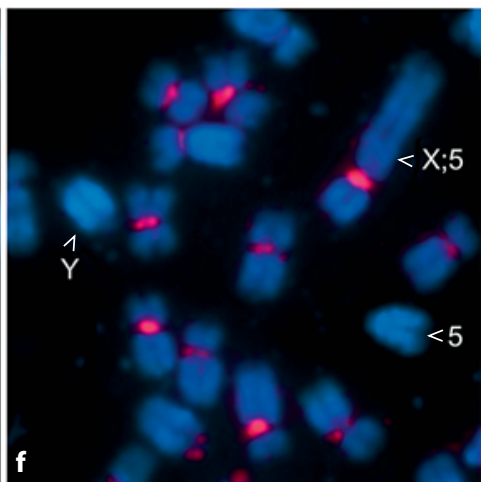

Fig. 5. In situ hybridization of the species-specific sat I (green) and sat II (red) probes to partial metaphase chromosomes of: a, d N. dama ruficollis, b, e G. leptoceros, c, f A. cervicapra. Centromeres of the unfused BTA 5 chromosomes are unlabeled in all 3 species. Bar $=5 \mu \mathrm{m}$. 
relied on G-banding to infer homology with cattle. Our results were in accordance with the previous findings, except for the identification of chromosome pairs $t(4 ; 29)$ and $\mathrm{t}(3 ; 28)$ in $N$. d. ruficollis, which on painting data correspond to $t(4 ; 28)$ and $t(6 ; 25)$. The chromosomes BTA3 and BTA29 are not involved in Robertsonian fusions. In G. leptoceros, Vassart et al. [1995] correctly documented the BTA homology for fusions with the exception of 5 pairs: $t(2 ; 6), t(3 ; 4), t(18 ; 25), t(22 ; 29)$ and $t(26 ; 27)$ which on painting data correspond to $\mathrm{t}(2 ; 3), \mathrm{t}(4 ; 6), \mathrm{t}(18 ; 29), \mathrm{t}(22 ; 25)$ and $t(26 ; 28)$, respectively. An earlier analysis of the $A$. cervicapra karyotype (female, $2 \mathrm{n}=30, \mathrm{XX}$ ) was undertaken by Gallagher and Womack [1992] and relied on Q-banding. Our results are in accordance with their findings except for the identification of chromosome pairs $t(1 ; 29)$ and $\mathrm{t}(2 ; 25)$ which on painting data correspond to pairs $\mathrm{t}(1 ; 25)$ and $t(2 ; 29)$, apparently because the BTA25 and BTA29 were switched in the old standard nomenclature [see ISCNDB 2000 for explanation]. Another study of $A$. cervicapra of unknown origin (male, $2 \mathrm{n}=31, \mathrm{XY}$ ) using Gbanding was carried out by Vassart et al. [1995]. Our results differ from theirs in several aspects. Apart from the already mentioned reversion of the chromosomes BTA25 and BTA29, our results disagree with the identification of the cattle equivalent chromosomes BTA3, BTA4 and BTA6. Fusions $t(3 ; 19), t(4 ; 24)$ and $t(6 ; 27)$ were identified by painting probes as $\mathrm{t}(4 ; 19), \mathrm{t}(6 ; 24)$ and $\mathrm{t}(3 ; 27)$. The examined animal was homozygous for the $\operatorname{rob}(8 ; 14)$ fusion. This fusion in the homozygous state was also described by Gallagher and Womack [1992]. The G-banded karyotype of A. cervicapra (female, $2 \mathrm{n}=32, \mathrm{XX}$ ) published in O'Brien et al. [2006] identified a double heterozygote for $t(8 ; 14)$ and $t(21 ; 26)$. One of the 4 animals examined in the present study was heterozygous for $t(8 ; 14)$, whilst in the other 3 , BTA 8 and BTA14 chromosomes were not fused. Centric fusion polymorphism appears to be quite common in the Bovidae family, but because most bovid cytogenetic data are derived from species long-bred in captivity, the situation in wild populations remains unknown [see, for example, Gallagher and Womack, 1992; Rubes et al., 2007; Pagacova et al., 2011]. These heterozygotes may be a result of hybridization in captivity between individuals representing chromosomally different wild populations [Kingswood et al., 1998; Pagacova et al., 2011].

\section{$X$ Chromosomes}

Chromosomal evolution in the studied clade has been prominently preceded by both Robertsonian and sex-toautosome translocations [Effron et al., 1976]. The compound sex-chromosomal configuration, i.e. X;A, Y;A and both X;A/Y;A translocation forms, although not common among mammals, occur frequently among bovid species [Rubes et al., 2008 in Tragelaphinae; Gallagher et al., 1998 in Boselaphini; Vassart et al., 1995 in gazelles; Effron et al., 1976 and Gallagher and Womack, 1992 in Antilopinae]. All modifications of the X chromosome, especially $\mathrm{X}$-autosome translocations, are prospectively powerful reproductive isolating mechanisms due to their impact on sex determination and/or on the X-inactivation process [King, 1993].

It is thought that (1) to prevent spreading of the $\mathrm{X}$ inactivation into the adjacent autosomal element and to avoid the silencing of autosomal genes, and (2) to allow for independent regulation of replication timing of the sex and autosomal segments, interstitial heterochromatic barriers are critical for the viability of these rearrangements in mammals [reviewed by Dobigny et al., 2004]. We explored this possibility in A. cervicapra, N. d. ruficollis and G. leptoceros, using PCR with SI and SII primer pairs designed from species-specific sat I and sat II DNA sequences. In spite of the fact that the early cytogenetic study of Vassart [1994] suggested no evidence of interstitial heterochromatic barriers using G-, C-, and R-banding in gazelles, our study using more modern techniques, clearly documented the centromeric heterochromatin occurrence in N. $d . r u$ ficollis (detectable by PCR and FISH) and G. leptoceros in the X;BTA5 junction which, although not highly amplified, might fulfill the role of chromatin insulator which separates the autosomal and sex chromosome compartments in X;A translocations. However, neither the PCR technique nor FISH provided evidence of repetitive DNA in A. cervicapra at the X;BTA5 junction. This prompts the question of whether there is another repetitive motif at the junction in this species detectable by distinct primers or whether different epigenetic pathways have been evolved to be responsible for maintaining an efficient isolation between sex and autosomal compartment in A. cervicapra. This finding requires further investigation.

It was concluded that 3 structural variants of the $\mathrm{X}$ chromosome have evolved among bovids: one submetacentric in morphology represented by cattle and 2 acrocentric types - eland and suni - characterized by Robinson et al. [1997, 1998]. All the species included in our study possess the $\mathrm{X}$ chromosome which can be classified as 'suni acrocentric type' (also referred to as caprine type) with a shift in centromere position in A. cervicapra and $N$. d. ruficollis. The centromere relocation in N.d.ruficollis disrupts the chromosomal material, but the ancient caprine-like X chromosome arrangement is still retained. Such intrachromosomal rearrangements of the sexual 
part of the X chromosome are supposed to be happened in N. granti and N. soemmerringii [Vassart et al., 1995].

For a clade that includes Antilope, Gazella, Nanger and Eudorcas, X;BTA5 translocation is a synapomorphy. Nevertheless, our results based on region-specific paints allowed us to discover that different chromosomal fusions (tandem in G. leptoceros and N. d. ruficollis, unlike centric fusion in A. cervicapra) assembled the 2 chromosomes. These findings raise an interesting question. Are these really tandem/centric fusions or have the species undergone chromosomal reorganization in chromosomal evolution, i.e. inversion of the sexual part of the X chromosome, most likely in A. cervicapra? In future, the examination of the $\mathrm{X}$ chromosome of Eudorcas members (E. rufina, rufifrons or thomsoni) may help to explain the rearrangements in the clade. Regrettably, material of these species was not available during our study.

During the course of $\mathrm{X}$ chromosome evolution in the studied species, addition/deletion of constitutive heterochromatin forming their Xp arms has evolved. While some species ( $G$. dorcas) have an acrocentric type of a compound X chromosome with minute Xp arms, other species, for example, G. leptoceros, E. thomsoni and A. cervicapra have a submetacentric type of compound X chromosome with short Xp arms [Effron et al., 1976; Vassart et al., 1995]. Furthermore, in the latter 2 species, polymorphic variations due to the differences in the quantity of the heterochromatic Xp arms have been reported in 2 female specimens [Effron et al., 1976]. We may suppose that the same repetitive DNA family of the Xp arms emerged in X chromosomes in the clade formed by Antilope, Eudorcas, Gazella and Nanger. Further studies, of course, are needed to confirm this assumption in the species which were not included in this study.

\section{NORs}

The use of silver staining may lead to errors in determining the number of NORs and their chromosomal positions due to their inability to detect transcriptionally inactive rRNA genes and the non-specific binding of silver nitrate with chromatin [Nguyen et al., 2008]. We attempted to construct a specific probe to precisely detect the rDNA locations using a FISH-based approach in the studied species. The studies of Gallagher et al. [1999] and Nguyen et al. [2008] indicated that a thorough understanding of these chromosomal structures may be useful for additional phylogenetic information concerning the Bovidae. The numbers of NORs reported here were obtained from a single specimen per species. All the species share the presence of NORs on BTA5 with Caprini, Bose- laphus and Tragelaphini [Nguyen et al., 2008]. On the other hand, NORs on chromosome BTA3 documented frequently in Bovidae, with the exception of Pseudoryx, Boselaphus [Nguyen et al., 2008] and Tetracerus [Ropiquet et al., 2010] known to date, were not identified in $N$. d. ruficollis.

\section{Satellite I and II DNA Sequences}

About one-fourth of the bovine genome comprises highly repeated DNA families, including both tandem satellite arrays and dispersed repeats [Modi et al., 1996]. Satellite sequences are mostly located in the centromeric and pericentric heterochromatin, i.e. in 2 epigenetically determined regions responsible for correct pairing and disjunction of eukaryotic chromosomes during cell division [reviewed by Plohl et al., 2008]. In our study, satellite sequences isolated from 3 Antilopini members representing the main motif of these repetitive DNAs were classified into 2 satellite types by sequence analysis. The first one (sat I) had approximately $790 \mathrm{bp}$ in all 3 species and contained the imperfect variants of $\mathrm{G}+\mathrm{C}$-rich 31-bp motif sequences as described in the bovine sat I DNA characterized by Plucienniczak et al. [1982]. In the second one, similarly to the published sat II DNA of Bovidae [Buckland, 1985], no internal repetition was found in the studied species. The length of repeat units (each about $700 \mathrm{bp}$ ) is very similar to the published sat II DNA (700 bp). Moreover, the GenBank BLASTN searches indicated that the sat II DNAs determined in our study showed $73 \%$ sequence homology with the sheep (Ovis aries) sat II DNA (GenBank: U24092). The chromosomal distribution of the satellite DNAs was examined by FISH. In A cervicapra and G. leptoceros, both sat I and II were localized in the centromeric regions, while in $N$. $d$. ruficollis, different distributions of the 2 repetitive DNA families in the centromeric regions have been revealed. This phenomenon is not uncommon within Bovidae. A previous report by D'Aiuto et al. [1997] described the distribution of sat I and sat II organized in 2 separate domains in the centromeric region in sheep (Ovis aries). More recently, Cernohorska et al. [2011] revealed 2 different hybridization patterns with centromeric clones in Kirk's dik-dik (Madoqua kirkii, Neotragini). Two satellite DNA families organized in 2 separate domains located in centromeric regions are well-known in the mouse genome as well [Kipling et al., 1991]. As far as we are aware, most of the repetitive families are concentrated in the centromeric region of Bovidae. Less frequently, sat I occupies the centromeric regions of chromosomes, while sat II occupies the pericentric ones. Rarely, if ever, the distribution is the 
है

BTA5

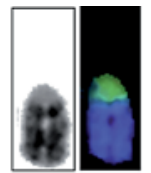

BTA14

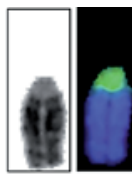

BTA8

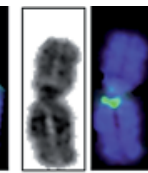

BTA $8 ; 14$
Fig. 6. Different chromosomes detected in A. cervicapra showing hybridization patterns using sat I DNA probe. The centromere of the unfused BTA5 is unlabeled. In each example, G-banded chromosomes are on the left.

same as described in N. d. ruficollis, i.e. sat II is located in centromeric areas and sat I in pericentric ones. In spite of the progressive accumulation of data, the functional significance of satellite DNAs arranged in long tandem arrays is still mostly unknown. It is assumed that centromeres in most eukaryotes are determined epigenetically rather than by primary DNA sequence [Sullivan et al., 2001; Malik and Henikoff, 2002].

\section{Is the Centromere of the BTA5 Ortholog Protected} against Prospective Fusions?

The subject of many current investigations in diverse eukaryotes is the contribution of both genetic and epigenetic determinants to the centromere function. In our investigation, the centromere of BTA5 orthologs in males (Y2, an ancestral autosome) in the XY1Y2 sex chromosomal system demonstrated particular need for more detailed discussion. Our FISH experiments with sat I and II probes resulted in no labeling of the unfused BTA5 centromere in A. cervicapra, G. leptoceros and N.d. ruficollis containing X;BTA5 elements. As it is apparently seen in A. cervicapra (fig. 5c, 6), the unfused BTA8 and BTA14 chromosomes involved in centric fusion polymorphism in that species show a strong fluorescent signal in the centromeric region, unlike the centromere of BTA5. On Gbanded chromosomes, the BTA5 in all 3 species was found to possess a markedly lesser, dark-banded centromere in comparison with other acrocentric chromosomes (fig. 6) suggesting a lower number of centromeric DNA copies. Firstly, we assumed that this fact could exert an impact on the detection of centromeric DNA by FISH approaches. It is noteworthy that when we had to reuse the slides of $N$. d. ruficollis due to limited availability of chromosomal material, faint signals were detected on the BTA5 using both sat I and sat II probes (see fig. 3). It seems that repeated FISH treatment made chromosomal DNA accessible to hybridization procedures. The question arises as to whether the BTA5 centromere is more condensed or epigenetically protected from prospective fusions with other acrocentrics to avoid potentially severe consequences of such rearrangements for fertility. In this context, it would be worth mentioning that in greater kudu (Tragelaphus strepsiceros), exhibiting Y;BTA13 translocation (X1X2Y sex chromosome system), the unfused BTA13 has a large heterochromatin block in the centromeric region, which is absent in the chromosome Y;BTA13 [Rubes et al., 2008]. This fact supports our hypothesis that the centromeres of chromosomes involved in the X;A sex determination system, i.e. the unfused A chromosomes, might be more protected. However, further studies of this topic are necessary for verification of this hypothesis.

\section{Conclusion}

The main task in this study was a detailed comparison of chromosomal homologies among the X;BTA5 compound chromosomes of 3 species represented by the $A n$ tilope, Gazella, Nanger and Eudorcas clade. Although it is not easy to interpret the findings within phylogenetic context (i.e. independent fusions or inversions of the sexual part of X chromosomes), we assume that all modifications of $\mathrm{X}$ chromosomes have evolved in the species as a powerful reproductive isolating mechanism before numerous and different Robertsonian fusions had been fixed and accumulated in the clade (diploid chromosome numbers range between 30 and 58 in this clade). While this is only an assumption at this time, support comes from the chromosomal complement of E. rufifrons $(2 \mathrm{n}=$ 58) previously studied by Vassart et al. [1995] retaining the ancestral-like chromosomal complement consisting of 56 acrocentric autosomes, biarmed X;BTA5 and biarmed Y;BTA16 chromosomes. We speculate that largescale $\mathrm{X}$ chromosome rearrangements in the species-rich Bovidae family may act as a driving force in the course of evolution creating post-meiotic barriers between newly formed species.

\section{Acknowledgements}

This work was supported by grants GA CR P506/10/0421, GA CR P502/11/0719, MZE 0002716202 and by the project 'CEITEC - Central European Institute of Technology' (CZ.1.05/1.1.00/102.0068) from European Regional Development Funds. 


\section{References}

Ashley T: X-autosome translocations, meiotic synapsis, chromosome evolution and speciation. Cytogenet Genome Res 96:33-39 (2002).

Buckland RA: Sequence and evolution of related bovine and caprine satellite DNAs. J Mol Biol 186:25-30 (1985).

-Cernohorska H, Kubickova S, Vahala J, Robinson TJ, Rubes J: Cytotypes of Kirk's dik-dik (Madoqua kirkii, Bovidae) show multiple tandem fusions. Cytogenet Genome Res 132: 255-263 (2011)

Chaves R, Santos S, Guedes-Pinto H: Comparative analysis (Hippotragini versus Caprini, Bovidae) of X-chromosome's constitutive heterochromatin by in situ restriction endonuclease digestion: X-chromosome constitutive heterochromatin evolution. Genetica 121:315-325 (2004).

D'Aiuto L, Barsanti P, Mauro S, Cserpan I, Lanave C, Ciccarese S: Physical relationship between satellite I and II DNA in centromeric regions of sheep chromosomes. Chromosome Res 5:375-381 (1997).

Dobigny G, Durcoz JF, Robinson TJ, Volobouev $\mathrm{V}$ : Viability of X-autosome translocation in mammals: an epigenomic hypothesis from a rodent case-study. Chromosoma 113:34-41 (2004).

-Effron M, Bogart M, Kumamoto A, Benirschke $\mathrm{K}$ : Chromosome studies in the mammalian subfamily Antilopinae. Genetica 46:419444 (1976).

-Gallagher DS, Womack JE: Chromosome conservation in the Bovidae. J Hered 83:287-298 (1992).

-Gallagher DS, Davis SK, De Donato M, Burzlaff JD, Womack JE, et al: A caryotypic analysis of nilgai, Boselaphus tragocamelus (Artiodactyla: Bovidae: Bovinae). Chromosome Res 6:505-513 (1998).

-Gallagher DS, Davis SK, De Donato M, Burzlaff JD, Womack JE, et al: A molecular cytogenetic analysis of the tribe Bovini (Artiodactyla: Bovidae: Bovinae) with an emphasis on sex chromosome morphology and NOR distribution. Chromosome Res 7:481-492 (1999).

-Goodpasture C, Bloom SE: Visualization of nucleolar organizer in mammalian chromosomes using silver staining. Chromosoma 53:37-50 (1975).
Iannuzzi L, Di Meo GP, Perucatti A, Incarnato D, Schibler L, et al: Comparative FISH mapping of bovid $\mathrm{X}$ chromosomes reveals homologies and divergences between the subfamilies Bovinae and Caprinae. Cytogenet Cell Genet 89:171-176 (2000).

ISCNDB 2000: International System for Chromosome Nomenclature of Domestic Bovids. Cytogenet Cell Genet 92:283-299 (2001).

King M: Species Evolution: The Role of Chromosome Change (Cambridge University Press, Cambridge, UK 1993).

Kingswood SC, Kumamoto AT, Charter SJ, Aman RA, Ryder OA: Centric fusion polymorphisms in waterbuck (Kobus ellipsiprymnus). J Hered 89:96-100 (1998).

-Kipling D, Ackford HW, Taylor BA, Cooke HJ: Mouse minor satellite DNA genetically maps to the centromere and is physically linked to the proximal telomere. Genetics 11:235-241 (1991).

Kubickova S, Cernohorska H, Musilova P, Rubes J: The use of laser microdissection for the preparation of chromosome-specific painting probes in farm animals. Chromosome Res 10:571-577 (2002).

Malik HS, Henikoff S: Conflict begets complexity: the evolution of centromeres. Curr Opin Genet Dev 12:711-718 (2002).

Modi WS, Gallagher DS, Womack JE: Evolutionary histories of highly repeated DNA families among the Artiodactyla (Mammalia). J Mol Evol 42:337-349 (1996).

-Nguyen TT, Aniskin VM, Gerbault-Seureau M, Planton H, Renard JP, et al: Phylogenetic position of the saola (Pseudoryx nghetinhensis) inferred from cytogenetic analysis of eleven species of Bovidae. Cytogenet Genome Res 122:41-54 (2008).

O'Brien SJ, Menninger JC, Nash WG: Atlas of Mammalian Chromosomes (John Wiley \& Sons, Inc., Hoboken, NJ 2006).

Pagacova E, Cernohorska H, Kubickova S, Vahala J, Rubes J: Centric fusion polymorphism in captive animals of family Bovidae. Conserv Genet 12:71-77 (2011).

Pauciullo A, Kubickova S, Cernohorska H, Petrova K, Berardino DD, et al: Isolation and physical localization of new chromosomespecific repeats in farm animals. Vet Med Czech 51:224-231 (2006).

-Plohl M, Luchetti A, Mestrovič N, Mantovani B: Satellite DNAs between selfishness and functionality: structure, genomics and evolution of tandem repeats in centromeric (hetero)chromatin. Gene 409:72-82 (2008).

-Plucienniczak A, Skowronski J, Jaworski J: Nucleotide sequence of bovine 1.715 satellite DNA and its relation to other bovine satellite sequences. J Mol Biol 158:293-304 (1982).
Robinson TJ, Harrison WR, Ponce de León FA, Elder FF: X chromosome evolution in the suni and eland antelope: detection of homologous regions by fluorescence in situ hybridization and G-banding. Cytogenet Cell Genet 77:218-222 (1997).

Robinson TJ, Harrison WR, Ponce de León FA, Davis SK, Elder FF: A molecular cytogenetic analysis of $\mathrm{X}$ chromosome repatterning in the Bovidae: transpositions, inversions, and phylogenetic inference. Cytogenet Cell Genet 80:179-184 (1998).

Robinson TJ, Ropiquet A: Examination of hemiplasy, homoplasy and phylogenetic discordance in chromosomal evolution of the Bovidae. Syst Biol 60:439-450 (2011).

Ropiquet A, Hassanin A, Pagacova E, GerbaultSeureau M, Cernohorska H, et al: A paradox revealed: Karyotype evolution in the fourhorned antelope occurs by tandem fusion (Mammalia, Bovidae, Tetracerus quadricornis). Chromosome Res 18:277-286 (2010).

Rubes J, Pagacova E, Kopecna O, Kubickova S, Cernohorska H, et al: Karyotype, centric fusion polymorphism and chromosomal aberrations in captive-born mountain reedbuck (Redunca fulvorufula). Cytogenet Genome Res 116:263-268 (2007).

Rubes J, Kubickova S, Pagacova E, Cernohorska $\mathrm{H}$, Berardino DD, et al: Phylogenomic study of spiral-horned antelope by cross-species chromosome painting. Chromosome Res 16: 935-947 (2008).

Seabright M: A rapid banding technique for human chromosomes. Lancet 2:971-972 (1971).

Sullivan BA, Blower MD, Karpen GH: Determining centromere identity: cyclical stories and forking paths. Nat Rev Genet 2:584-596 (2001).

Sumner AT: A simple technique for demonstrating centric heterochromatin. Exp Cell Res 75:304-306 (1972).

Vassart M: Evolution et diversité génétique chez les gazelles: appors de l'electrophorèse des protéines, de la cytogénétique et des microsatellites (PhD dissertation) (Orsay University, Paris 1994).

Vassart M, Seguela A, Hayes H: Chromosomal evolution in gazelles. J Hered 86:216-266 (1995).

Verma RS, Babu A: Human Chromosomes. Manual of Basic Techniques (Pergamon, New York 1989). 